\title{
ERRORS IN THE ESTIMATION OF ARTERIAL WALL SHEAR RATES THAT RESULT FROM CURVE FITTING OF VELOCITY PROFILES
}

\author{
Zheng Lou,* Wen-Jei Yang $†$ and Paul D. Stein $\ddagger$ \\ * Transportation Research Institute, University of Michigan. Ann Arbor, MI 48109, U.S.A.; $\dagger$ Mechanical \\ Engineering and Applied Mechanics, University of Michigan, Ann Arbor, MI 48109. U.S.A.; and $\ddagger$ Henry \\ Ford Heart and Vascular Institute, Detroit, MI 48202, U.S.A.
}

\begin{abstract}
An analysis was performed to determine the error that results from the estimation of the wall shear rates based on linear and quadratic curve-fittings of the measured velocity profiles. For steady, fully developed flow in a straight vessel, the error for the linear method is linearly related to the distance between the probe and the wall, $\mathrm{d} r_{1}$, and the error for the quadratic method is zero. With pulsatile flow, especially a physiological pulsatile flow in a large artery, the thickness of the velocity boundary layer, $\delta$ is small, and the error in the estimation of wall shear based on curve fitting is much higher than that with steady flow. In addition, there is a phase lag between the actual shear rate and the measured one. In oscillatory flow, the error increases with the distance ratio $\mathrm{d} r_{1} / \delta$ and. for a quadratic method, also with the distance ratio $\mathrm{d} r_{2} / \mathrm{d} r_{1}$, where $\mathrm{d} r_{2}$ is the distance of the second probe from the wall. The quadratic method has a distinct advantage in accuracy over the linear method when $\mathrm{d} r_{1} / \delta \ll 1$, i.e. when the first velocity point is well within the boundary layer. The use of this analysis in arterial flow involves many simplifications, including Newtonian fluid, rigid walls, and the linear summation of the harmonic components, and can provide more qualitative than quantitative guidance.
\end{abstract}

\section{INTRODUCTION}

An assessment of shear stress at the wall of arteries is important in the determination of whether fluiddynamic factors may contribute to the initiation or worsening of atherosclerotic lesions. There are three approaches toward the evaluation of the wall shear rate. The first is to measure the shear rate exactly on the wall surface using various methods: opaque coating layers (Adamson and Roach, 1981); electrochemical probes (Lutz et al., 1979; Talbot and Steinert, 1987; Yamaguchi and Hanai, 1988); and hot-film probes (Bellhouse and Schultz, 1966). These make use of the similarity between velocity and thermal or concentration boundary layers. With proper calibration and flush positioning, the measurements are accurate with steady or quasi-steady flow. The frequency response, however, with some of those methods (especially opaque coating), does not meet the demand required with pulsatile flow in large arteries (Pedley, 1980: Talbot and Steinert, 1987).

The second approach is to calculate the wall shear rate using the velocity profile derived from a nonlinear theory or wave equation and pressures measured at two neighboring stations (Ling and Atabek, 1972) or a flow rate. It is, however, difficult to monitor simultaneously the dynamic pressures at two sites. It is also not easy to measure accurately a dynamic flow rate in vivo. The theoretical derivation is complex, and this approach is even more complex for sites other than those in a straight tube. In the case of a bifurcation, for example, a full-scale theoretical analysis or

Accepted in final form 4 November 1992. simulation is required, using these measured data as the boundary conditions.

The third approach is to calculate the wall shear rate using point velocity measured at desired sites. To accomplish this, it is required that (a) the measuring sites are close to the wall surface, (b) the probe has a good frequency response, and (c) the probe does not disturb the flow. The fundamental instrumentation employed in the first approach may be used in velocity measurement (Ling et al., 1968). Laser Doppler Velocimeters (LDVs) have been widely used because of their noninvasiveness and wide bandwidth (Einav et al., 1975, 1990; Friedman and Deters, 1987; Friedman et al., 1981; Ku et al., 1985; Mark et al., 1989: Walburn and Stein, 1982). They have been used even for in vivo velocity measurements (Kajiya et al., 1985). The near-wall spatial resolution of most LDVs is about $0.5-0.9 \mathrm{~mm}$ because of the size of the measuring volume and optical interference from the wall (Friedman and Deters, 1987: Friedman et al. 1981). This is a large size, compared with the boundary layer thickness of pulsatile flow in a large artery, as we will show in the following discussion.

In any given experiment, there may be various errors, including those from the operator, instrument. and methodology. Any errors in velocity and position also influence the estimated values of shear rate. Precise localization of the arterial wall is not easy, especially with distensible walls. With a finite measuring volume, the LDV does not measure velocity precisely at a point. This is a serious problem in a narrow region with a large velocity gradient. The current analysis is concerned with errors that result from extrapolating to the arterial wall a measured profile of 
velocity with a curve-fitting process. This is a particular problem under the conditions of arterial flow (Lou and Yang, 1992).

\section{METHODS TO ESTIMATE WALL SHEAR RATES}

Many methods exist for estimating the wall shear rate from a polynomial velocity profile that fits the measured point velocities in arteries. The shear rate is, therefore, not obtained in a truly direct fashion. The experimentally obtained data must be extrapolated. The method of extrapolation can be classified according to the degree of polynomials assumed in the curve fitting of the velocity profiles. The simplest of all, called the linear method, is to measure the velocity component parallel to the wall, $u_{1}$, at a point that is a distance $\mathrm{d} r_{1}$ normal to the wall, and calculate the wall shear rate with the following equation (Duncan et al., 1990; Friedman et al., 1981; Mark et al., 1989):

$$
\dot{\gamma}_{\mathrm{w} f}=\frac{u_{1}}{\mathrm{~d} r_{1}} .
$$

This implicitly assumes a linear velocity distribution. This and all other methods assume that the fluid is nonslip at the wall and that the velocity at the probe is roughly parallel to the wall.

The second method, called the quadratic method, assumes a quadratic velocity distribution (Duncan et al., 1990; Ku et al., 1985). It needs an additional velocity $u_{2}$ at another point, distant $\mathrm{d} r_{2}$ from the wall. The second point is farther away from the wall than the first point. The wall shear rate is then calculated as

$$
\dot{\gamma}_{\mathrm{wq}}=\frac{u_{2} \mathrm{~d} r_{1}^{2}-u_{1} \mathrm{~d} r_{2}^{2}}{\mathrm{~d} r_{2} \mathrm{~d} r_{1}^{2}-\mathrm{d} r_{1} \mathrm{~d} r_{2}^{2}}
$$

In another method, a third velocity is measured at a point between the two points used in the quadratic method (Duncan et al., 1990; Ku et al., 1985). The least-squares method is adopted to accommodate all three points, or four points if the point at the wall is included, in a parabolic velocity profile. The benefit of the extra point is doubtful because it forces the velocity profile to comply more to the data in the region farther away from the wall than to the data closer to the wall.

Higher-order polynomial velocity profiles have also been used in the aortic bifurcation study (Walburn and Stein, 1982). A higher-order polynomial, however, is generally less robust. As the order of the curve fitting increases, the result becomes sensitive to smallamplitude, more-or-less randomly distributed errors in the data (Roache, 1972).

Analytical solutions can readily be obtaincd for simplified steady and oscillatory flows. Flow in arteries is nonlinear and complex, and it is simplified in the current analysis to capture the major characteristics of the flow. Two assumptions are made: (1) blood is assumed to be Newtonian; and (2) the arterial walls are assumed to be rigid. Blood is assumed to be
Newtonian because its non-Newtonian behavior does not change significantly the wall shear stress in large arteries (Lou and Yang, 1993b; Perktold et al., 1991). The non-Newtonian effect on the error analysis will be, at most, of the second order. Also, most in vitro experiments and numerical simulations employed Newtonian fluids. The arterial walls are assumed to be rigid because the effect of the wall distensibility on the flow field is relatively small (Deters et al., 1986; Lou and Yang, 1993a).

A steady, fully developed flow in a straight tube is considered first. Its velocity profile and wall shear rate can be described by

$$
u^{*}=1-r^{* 2}
$$

and

$$
\frac{\dot{\gamma}_{w}}{u_{\max } / r_{0}}=-2
$$

with

$$
\begin{aligned}
& r^{*}=\frac{r}{r_{0}}, \\
& u^{*}=\frac{u}{u_{\max }},
\end{aligned}
$$

where $r$ is radial position from the axis in a cylindrical coordinate system, $r_{0}$ the radius of the vessel, $u$ the velocity at $r, u_{\max }$ the velocity at the center, and $\dot{\gamma}_{\mathrm{w}}$ the shear rate at the wall.

For a flow induced by an oscillatory pressure gradient whose amplitude is equal to the pressure gradient amplitude of the previous steady Poiseuille flow and whose angular frequency is $\omega$, the approximate analytical solution is (White, 1974)

$$
u^{*}=\frac{4}{\alpha^{2}}\left[\sin \theta-\frac{\mathrm{e}^{-B}}{\sqrt{r^{*}}} \sin (\theta-B)\right] \text { for } \alpha>2,
$$

with

$$
\begin{aligned}
& B=\left(1-r^{*}\right) \frac{\alpha}{\sqrt{2}}, \\
& \alpha=r_{0} \sqrt{\frac{\omega}{v}}, \\
& \theta=\omega t,
\end{aligned}
$$

where $\alpha$ is the Womersley number, $t$ the time, $\theta$ the phase angle within a cycle, and $v$ the kinematic viscosity. The exact solution for velocity is in the form of the Bessel function of the first kind and of zero order (Sexl, 1930), from which equation (7) is derived using the asymptotic expansion. The error in equation (7) itself is of the order of $1 / \alpha^{4}$. With a physiological $\alpha$ ranging from 5 to 10 in large arteries, the error will be $0.16 \%$ or less. Equation (7) provides a simple solution to the problem, without causing any practical error.

The same $u_{\max }$ that appeared in the steady flow is used in equation (7) to obtain the dimensionless velo- 
city $u^{*}$. The peak value for $u^{*}$ in equation (3) is 1 . The amplitude of $u^{*}$ in equation (7) is generally smaller than one because a large portion of the same pressure gradient amplitude is consumed in balancing the inertia force in the oscillatory flow.

From equation (7), the velocity gradient is derived as

$$
\begin{gathered}
\frac{\hat{\partial u^{*}}}{\partial r^{*}}=-\frac{4}{\alpha \sqrt{2 r^{*}}} \mathrm{e}^{-B}\left[\left(1-\frac{1}{\sqrt{2} \alpha r^{*}}\right)\right. \\
\sin (\theta-B)+\cos (\theta-B)] .
\end{gathered}
$$

The shear rate at the wall, $\dot{\gamma}_{\mathrm{w}}$, can be calculated from the above equation with $r^{*}=1$ and $B=0$, resulting in

$$
\frac{\dot{\gamma}_{w}}{u_{\max } / r_{0}}=-\frac{4}{\sqrt{2} \alpha}\left[\left(1-\frac{1}{\sqrt{2} \alpha}\right) \sin \theta+\cos \theta\right]
$$

With a large $\alpha$, there is a region of high-velocity flow near the wall, where the mean-square velocity is higher than that at the center line (White, 1974). An overshoot exists near the wall at $B=2.284$, where the boundary layer thickness, $\delta$, is defined and obtained as

$$
\delta=r_{0}-r_{B=2.284}=3.23 \sqrt{\frac{\gamma}{\omega}}=3.23 r_{0} / \alpha .
$$

For the wall shear rate in oscillatory flow to be accurate, the velocity probe must be placed well within the boundary layer and as close to the wall as possible. The relative position of the probe within the boundary layer can be expressed as

$$
\frac{\mathrm{d} r_{1}}{\delta}=0.31 \frac{\mathrm{d} r_{1}}{r_{0}} \alpha
$$

Errors from curve fitting are isolated by assuming accurate velocity and position data. Errors from curve fitting are 'hard' errors in a sense that they result from the scheme itself. They do not appear randomly and can be analyzed systematically. Errors from velocity and position data are 'soft' in a sense that they depend on many random factors, including calibrations, measuring procedures (like seeding and focusing in the LDV measurement), and data filtering. They are random both in phase and in amplitude. If there are errors of $+10 \%$ and $-10 \%$ in the velocity $\left(u_{1}\right)$, there will be soft errors of $+10 \%$ and $-10 \%$, respectively, in the wall shear rate resulting from the linear method [equation (1)]. If there are errors of $+10 \%$ and $-10 \%$ in the probe position $\left(\mathrm{d} r_{1}\right)$, the resulting soft errors in the wall shear rate will be about $-9 \%$ and $+11 \%$, respectively, for the linear method. These soft errors are additive and are also additive with hard errors. The influences of the soft errors to the total errors are not as straightforward in the case of the quadratic method [equation (2)]. It can generally be stated that the total errors become more sensitive to the soft errors when $\mathrm{d} r_{2}$ is close to $\mathrm{d} r_{1}$. The random- ness in the velocity and position data will introduce a certain amount of randomness to the overall accuracy. One can make multiple measurements and take their average to filter out the randomness in the data. As for the hard errors, i.e. those from curve fitting, one has to resort to analytical tools.

Analytical velocities are determined from equations (3) and (7) for steady and oscillatory flows, respectively. With the analytical velocities, the curve-fitting wall shear rates, $\dot{\gamma}_{\text {wef }}$ 's are calculated using equations (1) and (2) for the linear and quadratic methods, respectively. The analytical wall shear rates, $\dot{\gamma}_{w}$ 's, are obtained from equations (4) and (12) for steady and unsteady flows, respectively. The amount of the wall shear rate underestimated is determined as follows:

amount underestimated $=\frac{\left|\dot{\gamma}_{w c r}\right|-\left|\dot{\gamma}_{w}\right|}{\left|\dot{\gamma}_{w}\right|} \times 100 \%$.

\section{RESULTS}

For steady, fully developed flow in a straight cylindrical pipc, the linear method consistently underestimates the wall shear rate. The error is linearly related to $\mathrm{d} r_{1}$ (Fig. 1). In the case of the quadratic method with the distance ratio $\mathrm{d} r_{2} / \mathrm{d} r_{1}=2$, the curve-fitting and analytical solutions are identical as expected in a parabolic velocity. The same should be true with other values of the distance ratio $\mathrm{d} r_{2} / \mathrm{d} r_{1}$ because of the parabolic nature of the velocity.

For oscillatory flow in a straight cylindrical pipe, the linear method produces a shear rate profile of a similar trend as the analytical solution (Fig. 2). However, the linear method underestimates the amplitude of the wall shear rate. The curve-fitting shear rate peaks at a phase angle lagging behind that of the analytical solution. The phase lag causes the curve fitting to overestimate the shear rate at certain periods of a cycle, namely, at phase angles $>100^{\circ}$. The error in the amplitude (Fig. 3) increases both with $\mathrm{d} r_{1} / r_{0}$ and with $\alpha$, and so does the phase lag (Fig. 4). With the

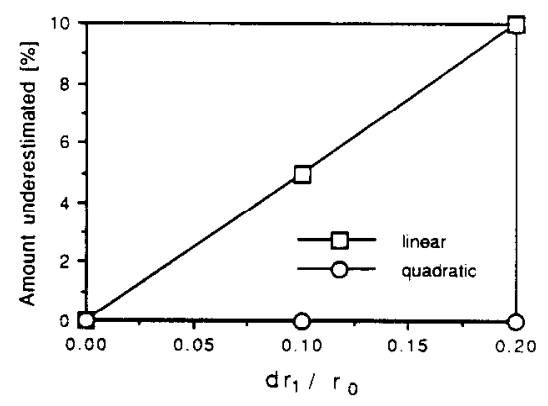

Fig. 1. The error that results from an estimation of the wall shear rate using linear and quadratic (with $\mathrm{d} r_{2}=2 \mathrm{~d} r_{1}$ ) curve fittings of the velocity profile for steady, fully developed flow in a straight pipe. The linear method consistently underestimates the wall shear rate, while the quadratic method generates exact solutions. 


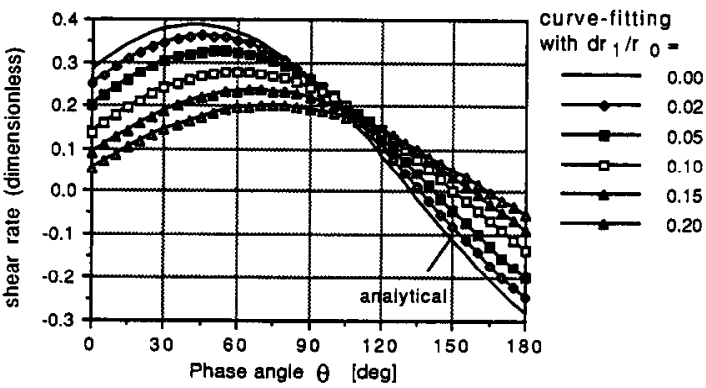

Fig. 2. A comparison of wall shear rates calculated from an analytical solution and obtained by curve fittings with the linear method. Flow was oscillating $(\alpha=10)$ in a straight cylindrical pipe. All the shear rates were nondimensionalized, by dividing by $u_{\max } / r_{0}$. The linear method underestimates the amplitude of the wall shear rate. The curve-fitting shear rate peaks at a phase angle lagging behind that of the analytical solution.

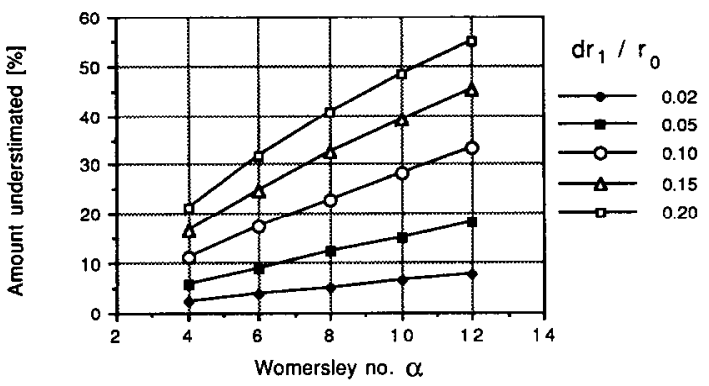

Fig. 3. The error in the peak wall shear rate as a function of $x$ (the Womersley number) and $\mathrm{d} r_{1} / r_{0}$ (the ratio of the distance beween the velocity probe and the arterial wall to the radius of the artery), obtained by the linear method, with oscillating flow in a straight cylindrical pipe. The error in the peak wall shear rate grows with both $\mathrm{d} r_{1} / r_{0}$ and $\alpha$.

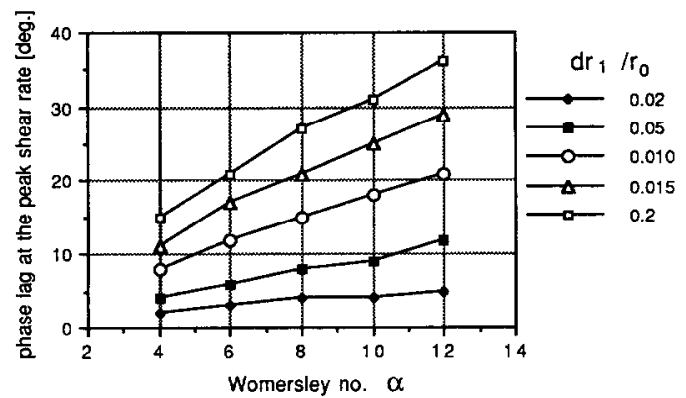

Fig. 4. The phase lag at the peak wall shear rate as a function of $\alpha$ (the Womersley number) and $\mathrm{d} r_{1} / r_{0}$ (the ratio of the distance between the velocity probe and the arterial wall to the radius of the artery), obtained by the linear method, with oscillating flow in a straight cylindrical pipe. The error in the phase lag grows with both $\mathrm{d} r_{1} / r_{0}$ and $\alpha$.

help of equation (14), two independent variables $\mathrm{d} r_{1} / r_{0}$ and $\alpha$ are combined into a variable $\mathrm{d} r_{1} / \delta$, which singularly determines the error amplitude (Fig. 5) and phase lag (Fig. 6). The relationships in

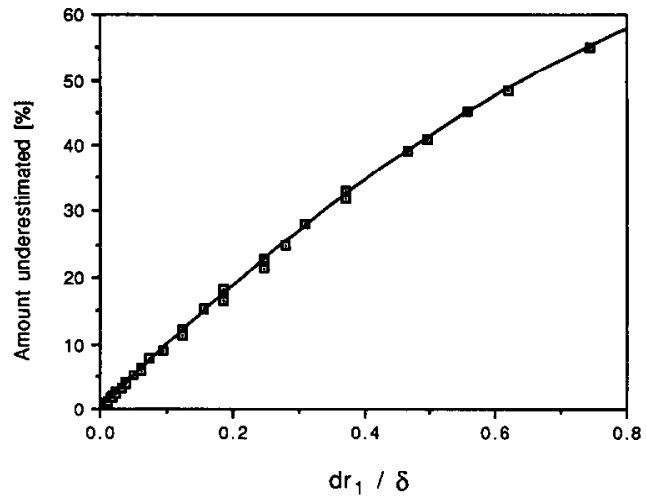

Fig. 5. The error in the peak wall shear rate versus $\mathrm{d} r_{1} / \delta$ (the ratio of the distance between the velocity probe and the arterial wall to the boundary layer thickness), obtained by the linear method, with oscillating flow in a straight cylindrical pipe. The error increases with a single variable $\mathrm{d} r_{1} / \delta$.

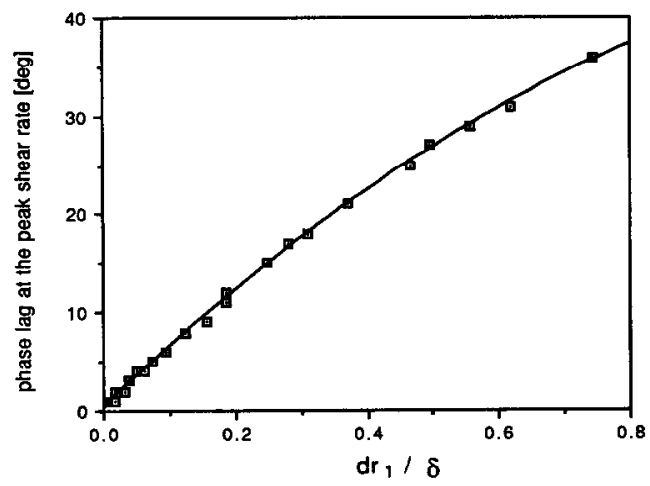

Fig. 6. The phase lag at the peak wall shear rate versus $\mathrm{d} r_{1} / \delta$ (the ratio of the distance between the velocity probe and the arterial wall to the boundary layer thickness), obtained by the linear method, with oscillating flow in a straight cylindrical pipe. The phase lag increases with a single variable $\mathrm{d} r_{1} / \delta$.

Figs 5 and 6 can be algebraically summarized as follows:

amount underestimated $(\%)$

$$
\approx 99.62\left(\mathrm{~d} r_{1} / \delta\right)-34.40\left(\mathrm{~d} r_{1} / \delta\right)^{2}
$$

and

phase lag at the peak shear rate $\left({ }^{\circ}\right)$

$$
\approx 63.98\left(\mathrm{~d} r_{1} / \delta\right)-22.23\left(\mathrm{~d} r_{1} / \delta\right)^{2} \text {. }
$$

The closer the probe is to the wall, relative to the boundary layer thickness, $\delta$, the more accurate is the extrapolated wall shear rate.

A similar trend is observed in the error amplitude (Fig. 7) and phase lag (Fig. 8) for the results obtained by the quadratic method, with the distance ratio $\mathrm{d} r_{2} / d r_{1}$ set at seven different values $(1.01,1.1$, $1.3,1.5,2.0,2.5$, and 3.0). When $\mathrm{d} r_{2} / \mathrm{d} r_{1} \leqslant 2.0$, the 


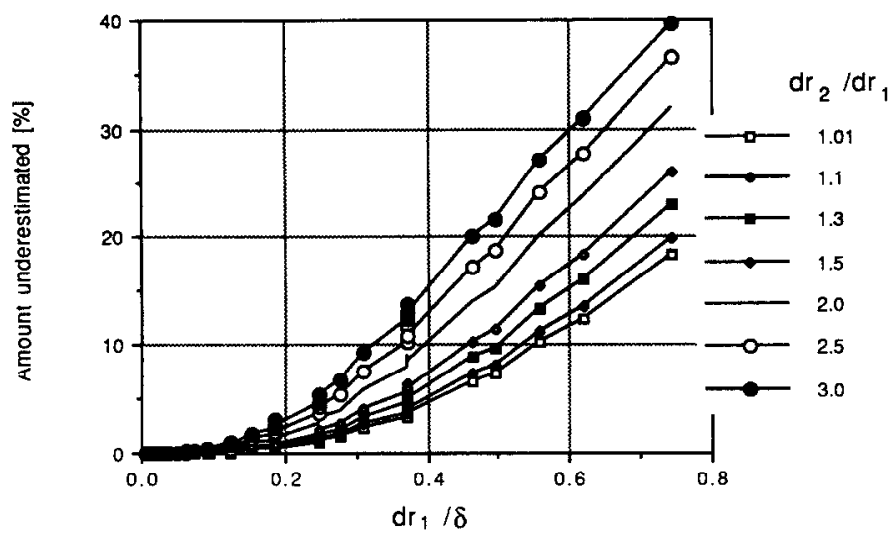

Fig. 7. The error in the peak wall shear rate versus $\mathrm{d} r_{1} / \delta$ (the ratio of the distance between the first velocity probe and the arterial wall to the boundary layer thickness), obtained by the quadratic method with $\mathrm{d} r_{2} / \mathrm{d} r_{1}$ (the ratio of the distance between the second velocity probe and the arterial wall to the distance between the first velocity probe and the wall) $=1.01 .1 .1,1.3,1.5 .2 .0,2.5$, and 3.0, with oscillating flow in a straight cylindrical pipe. At a fixed $\mathrm{d} r_{2} / \mathrm{d} r_{1}$, the error increases with $\mathrm{d} r_{1} / \delta$. At a fixed $\mathrm{d} r_{1} / \delta$, the error increases with $\mathrm{d} r_{2} / \mathrm{d} r_{1}$.

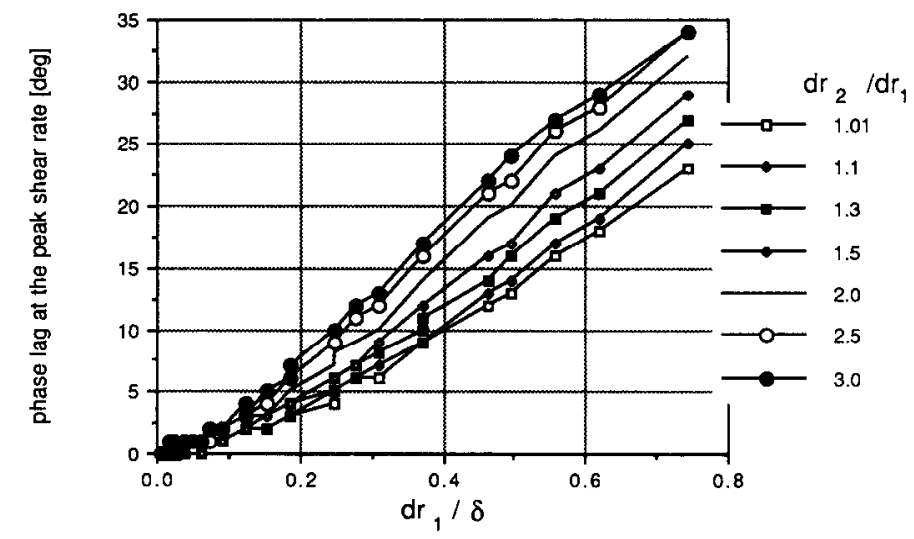

Fig. 8. The phase lag at the peak wall shear rate versus $\mathrm{d} r_{1} / \delta$ (the ratio of the distance between the first velocity probe and the arterial wall to the boundary layer thickness), obtained by the quadratic method with $\mathrm{d} r_{2} / \mathrm{d} r_{1}$ (the ratio of the distance between the second velocity probe and the arterial wall to the distance between the first velocity probe and the wall) $=1.01,1.1,1.3,1.5,2.0,2.5$, and 3.0, with oscillating flow in a straight cylindrical pipe. At a fixed $\mathrm{d} r_{2} / \mathrm{d} r_{1}$, the phase lag increases with $\mathrm{d} r_{1} / \delta$. At a fixed $\mathrm{d} r_{1} / \delta$, the phase lag increases with $\mathrm{d} r_{2} / \mathrm{d} r_{1}$.

quadratic method is generally more accurate both in the shear rate amplitude and in the phase, especially at small $\mathrm{d} r_{1} / \delta$, than the linear method. When $\mathrm{d} r_{1}$ approaches $\delta$, however, the accuracy of the quadratic method deteriorates much faster and, for cases with large $\mathrm{d} r_{2} / \mathrm{d} r_{1}$, may become worse than that of the linear method. At $\mathrm{d} r_{2} / \mathrm{d} r_{1}=2.0$,

amount underestimated $(\%) \approx 60.72\left(\mathrm{~d} r_{1} / \delta\right)^{2}$

and

phase lag at the peak shear rate $\left({ }^{\circ}\right)$

$$
\begin{array}{r}
\approx 29.61\left(\mathrm{~d} r_{1} / \delta\right) \\
+21.84\left(\mathrm{~d} r_{1} / \delta\right)^{2} .
\end{array}
$$

At a fixed $\mathrm{d} r_{1}$, errors increase with $\mathrm{d} r_{2}$. When the distance ratio $\mathrm{d} r_{2} / \mathrm{d} r_{1}=2$, the three points including the no-slip point at the wall are equally spaced. It is feasible to have $\mathrm{d} r_{2} / \mathrm{d} r_{1}<2$ because the distance between the two measuring points, $\mathrm{d} r_{2}-\mathrm{d} r_{1}$, is limited only by the spatial resolution of the LDV, and $\mathrm{d} r_{1}$ is restricted by the optical interference from the wall as well as the spatial resolution of the LDV. As the distance ratio $\mathrm{d} r_{2} / \mathrm{d} r_{1}$ approaches unity, the curvefitting error decreases. However, additional errors (or 'soft' errors) caused by inaccurate velocity and position data will increase substantially as $\mathrm{d} r_{2}-\mathrm{d} r_{1}$ approaches zero in equation (2).

The results indicate that errors are much higher with oscillatory flow than with steady flow. Errors from the linear (with $\mathrm{d} r_{1} / \delta=0.1$ ) and quadratic (with 
$\mathrm{d} r_{1} / \delta=0.1$ and $\left.\mathrm{d} r_{2} / \mathrm{d} r_{1}=2\right)$ methods for oscillatory flow with $\alpha=10$ are 28 and $6 \%$, respectively, and those for steady flow are only 5 and $0 \%$, respectively. The errors are different by an order of magnitude between the two flows.

Around arterial bends and bifurcation, one cannot directly apply the above results, which are derived for a straight pipe. The boundary layer thickness in human arteries varies because of the bends and bifurcations. At the aortic bifurcation, the boundary layer thickness around the vertex is thin, resulting in much higher errors than those in the asymptotic regions. Estimates of the boundary layer thickness can be made as follows for some conditions such as plug flow. Plug flow is typical in pulsatile flow in large arteries. A simple plug flow model is used to estimate the variation in boundary layer thickness from other parameters. The velocity in the core of the flow is assumed to be uniform at $u_{0}$. The velocity in the boundary is approximated with a parabolic velocity profile with the following boundary conditions: (a) no slip at the wall; (b) $u=u_{0}$ at the edge of the boundary layer; (c) zero velocity gradient at the edge of the boundary layer; and (d) a shear rate of $\dot{\gamma}_{w}$ at the wall. The resulting boundary layer thickness is $2 u_{0} / \dot{\gamma}_{w}$. If one assumes $u_{0}$ to be approximatcly cqual to the mean velocity and if the flow rate does not change with the axial location in a continuous artery (i.e. no bifurcation in the arterial segment of interest), $u_{0}$ is approximately proportional to the inverse of the cross section, $A$. Then one has

$$
\delta \propto \frac{1}{A \dot{\gamma}_{\mathrm{w}}}
$$

The velocity profile at a branch entrance is not as uniform as that in the asymptotic regions, and a minor deviation is expected when equation (20) is used. The local boundary layer thickness can be estimated as follows:

$$
\delta_{\text {local }} \approx \frac{\delta_{\text {asymptotic }}}{f_{\text {location }}},
$$

where $\delta_{\text {asymptotic }}$ is from equation (13) and $f_{\text {location }}$, the location correction factor, is defined as

$$
f_{\text {location }} \approx \frac{\left(A \dot{\gamma}_{\mathrm{w}}\right)_{\text {local }}}{\left(A \dot{\gamma}_{\mathrm{w}}\right)_{\text {asymptotic }}} .
$$

Obtaining $f_{\text {location }}$ from equation (22) demands the knowledge of $\dot{\gamma}_{w}$, whereas an accurate estimation of $\dot{\gamma}_{w}$ requires $f_{\text {location. }}$. A complete solution may need several iterations between $f_{\text {location }}$ and $\dot{\gamma}_{w}$. For the branch of the aortic bifurcation, the average value of $f_{\text {location }}$ is about 2-3 (Lou and Yang, 1991).

Physiological flow waves are not sinusoidal although the oscillatory waves used thus far in the analysis were sinusoidal. A physiologically pulsatile flow has a mean flow and many harmonic components. In a linear case, the total velocity is the summation of the mean and individual harmonic compon- ents. The sum of the first eight harmonics includes at least $99 \%$ of the variance of the original curves (Bergel and Milnor, 1965; McDonald, 1974), and the sum of the first two harmonics alone contains $50-70 \%$ of the total variance (Milnor, 1989). A complete error analysis for a physiological flow has to include both the mean and individual harmonic components.

An omission of the mean flow does not influence substantially the quality of the error analysis in large arteries. For a velocity profile around the aortic bifurcation, for example, the amplitudes of the mean and the first four significant harmonic components, normalized by the amplitude of the fundamental (or first) harmonic component, are $0.40,1.00,0.97,0.47$ and 0.14 (McDonald, 1974). The amplitude of the mean flow is relatively small in larger arteries. The portion of the shear stress contributed by the mean flow, a steady flow, is even smaller. For a typical aortic bifurcation, the shear stress induced by the mean flow alone is an order of magnitude smaller than that by the total physiological flow (Lou and Yang, 1991).

The Womersley number of the fourth harmonic is twice the fundamental Womersley number. The relative error of the fourth harmonic is slightly less than twice that of the fundamental harmonic obtained by the lincar method, but higher than twice that of the fundamental harmonic obtained by the quadratic method. All the four harmonics show a peak value near the peak of the flow pulsation. The second, third, and fourth harmonics contribute more than their shares of error at the peak because of their high Womersley numbers. Therefore, the estimation obtained from the fundamental Womersley number is a conservative one. An effective Womersley number, $\alpha_{\text {effective, may be used and is related to the funda- }}$ mental Womersley number, $\alpha_{\text {fund }}$, by

$$
\alpha_{\text {effective }}=f_{\text {wave }} \alpha_{\text {fund }} \text {. }
$$

Here $f_{\text {wave }}$ is a wave correcting factor that is the summation of the square root of the wave number weighted by the amplitude:

$$
f_{\text {wave }} \approx \frac{\sum_{n=1}^{\infty} A_{n} \sqrt{n}}{\sum_{n=1}^{\infty} A_{n}} .
$$

Around the aortic bifurcation, one obtains

$$
\begin{aligned}
& f_{\text {wave }} \\
& \qquad \frac{1+0.97 \times \sqrt{2}+0.47 \times \sqrt{3}+0.14 \sqrt{4}}{1+0.97+0.47+0.14}=1.343 .
\end{aligned}
$$

This linear summation is accurate in a linear case, but provides only an approximation in a general case. Considering the effects of the location and wave pattern, $\mathrm{d} r_{1} / \delta$ becomes

$$
\frac{\mathrm{d} r_{1}}{\delta}=f_{\text {location }} f_{\text {wave }}\left(0.31 \frac{\mathrm{d} r_{1}}{r_{0}} \alpha_{\text {fund }}\right) .
$$


For a typical branch of the aortic bifurcation that has a branch radius of $5 \mathrm{~mm}$, a kinematic viscosity of 4.5 centistokes, a fundamental frequency of $1 \mathrm{~Hz}$, a fundamental Womersley number of 6 , a $f_{\text {location }}$ of 2.5 , and a $f_{\text {wave }}$ of 1.343 , the linear and quadratic methods demand $\mathrm{d} r_{1}$ to be less than 0.04 and $0.24 \mathrm{~mm}$ (with $\mathrm{d} r_{2} / \mathrm{d} r_{1}=2$ ), respectively, for the curve-fitting errors to be less than $5 \%$.

With $\mathrm{d} r_{1}=0.5 \mathrm{~mm}$, a typical value in the current literature, $\mathrm{d} r_{1} / \delta$ is equal to 0.624 . In estimating the peak shear rate at the aortic bifurcation, the linear method may yield a curve-fitting error of $49 \%$ with a phase lag of $32^{\circ}$, while the quadratic method, with $\mathrm{d} r_{2} / \mathrm{d} r_{1}=2$, produces a curve-fitting error of $23 \%$ with a phase lag of $26^{\circ}$.

\section{DISCUSSION}

The above results show that the spatial resolution of a conventional LDV may not be adequate for the study of arterial flow. By designing a special device to focus laser beams into a small measuring volume $(2 \times 2 \times 10 \mu \mathrm{m})$, Einav et al. (1990) were able to measure the shear stress along the cusp of a tri-leaflet prosthetic valve. Velocity was measured at several points, in $1 \mu \mathrm{m}$ intervals, in the vicinity of the cusp.

Brech and Bellhouse (1973) found that the measured wall shear stress (using thin-film heated elements) was always higher than that extrapolated (using linear method) from the measured velocity data (using a thin-film velocity probe), by $1-45 \%$ depending upon the position within a bifurcation. In a branch of a diameter of $19 \mathrm{~mm}$, wall shear stress was measured at $0.2,1.6$, and 3.7 diameters downstream from the apex. The velocity probe was placed $1.25 \mathrm{~mm}$ away from the wall, resulting in a $\mathrm{d} r_{1} / r_{0}$ of 0.13 . Brech and Bellhouse (1973) did not specify when in a wave cycle, at which of the three locations, and under which flow (steady or unsteady) they obtained the $45 \%$ error. Also, no time history of flow was given for the unsteady flow. The fundamental Womersley number for the unsteady flow in the branch was 6.56, using the definition in equation (9). One obtains $\mathrm{d} r_{1} / \delta=0.66$ from equation (26) if the peak error was obtained in a branch at a location that was 0.2 diameters downstream from the apex of the bifurcation, where one assumes a location correction factor $\left(f_{\text {location }}\right)$ of 2.5 , and if the wave correction factor $\left(f_{\text {wave }}\right)$ is equal to unity (i.e. a sinusoidal flow). With $\mathrm{d} r_{1} / \delta=0.66$, the amplitude of the error from equation (16) is $50.8 \%$, which is reasonably close to their experimental value of $45 \%$.

In several studies of arterial bifurcations, the probe distance $\mathrm{d} r_{1}$ was relatively large, e.g. $0.58 \mathrm{~mm}$ (Friedman et al., 1981) and $0.51 \mathrm{~mm}$ (Duncan et al., 1990). Duncan et al. (1990) observed that the mean shear rate from the linear method, $\dot{\gamma}_{r}$, and that from the quadratic method, $\dot{\gamma}_{q}$, were approximately related in the following manner:

$$
\dot{\gamma}_{q}=-79.6+1.87 \dot{\gamma}_{r}
$$

In a study of the carotid bifurcation using a quadratic curve-fitting method (Ku et al., 1985), the distance $\mathrm{d} r_{1}$ (whose value was not available) was subject to an uncertainty of approximately one-half the optical sample volume $(1.08 \times 0.12 \mathrm{~mm})$. The error for steady flow was within $15 \%$ according to their own estimation (Ku et al., 1985). The analytical tool proposed in the current study can be utilized to make a careful evaluation of errors in related studies.

Precautions should be taken in numerical simulations of arterial flows. There are two sets of variables: primitive variables (velocity and pressure); and the stream function and vorticity. With the primitive variables, the wall shear rate is evaluated indirectly using velocity data resulting from the simulation, and $\mathrm{d} r_{1}$ is equivalent to the distance between the first computational node and wall. Assuming velocity itself to be accurate, the error analysis should be the same as that just carried out. With the stream function and vorticity, the wall shear rate is directly equal to the wall vorticity. The error will come only in the process of solving system equations, and the error analysis will be different. The principle, that nodes should be as close to the wall as possible, is the same.

The use of this analysis in arterial flow involves many simplifications, including Newtonian fluid, rigid walls, and the linear summation of the harmonic components. It can provide more qualitative than quantitative guidance. Quantitative results of this analysis may not be as relevant in the region of permanent low shear stress, e.g. near the outer wall of the carotid sinus, where nonlinear phenomena (like flow separation) prevail.

In conclusion, linear and quadratic curve-fitting techniques for extrapolation of velocity to the vessel wall, under conditions of steady flow in straight rigid vessels, permit an accurate or reasonably accurate assessment of wall shear. With pulsatile flow, particularly around bifurcations and bends, errors are introduced, causing the shear rate to be underestimated.

\section{REFERENCES}

Adamson, S. L. and Roach, M. R. (1981) Measurement of wall shear stress in a glass model renal bifurcation by a technique that monitors the rate of erosion of an opaque coating layer. Biorheology 18, 9-21.

Bellhouse, B. J. and Schultz, D. L. (1966) Determination of mean and dynamic skin friction, separation and transition in low speed flow with a thin-film heated element. J. Fluid Mech. 24, 379-400.

Bergel, D. H. and Milnor, W. R. (1965) Pulmonary vascular impedance in the dog. Circ. Res. 16, 401-415.

Brech, R. and Bellhouse, B. J. (1973) Flow in branching vessels. Cardiovasc. Res. 7, 593-600.

Deters, O. J., Bargeron, C. B., Mark, F. F. and Friedman, M. H. (1986) Measurement of wall motion and wall shear in a compliant arterial cast. J. Biomech. Engng 108, 355-358. 
Duncan, D. D., Bargeron, C. B., Borchardt, S. E., Deters, O. J., Gearhart, S. A., Mark, F. F. and Friedman, M. H. (1990) The effect of compliance on wall shear in casts of a human aortic bifurcation. J. Biomech. Engng 112, $183-188$.

Einav, S., Berman, H. J., Fuhro, R. L., DiGiovanni, P. R., Fridman, J. D. and Fine, S. (1975) Measurement of blood flow in vivo by laser Doppler anemometry through a microscope. Biorheology 12, 203-205.

Einav, S., Stolero, D., Avidor, J. M., Elad, D. and Talbot, L. (1990) Wall shear stress distribution along the cusp of a tri-leaflet prosthetic valve. J. Biomed. Engng 12, 1318.

Friedman, M. H. and Deters, O. J. (1987) Correlation among shear rate measures in vascular flows. J. Biomech. Engng 109, 25-26.

Friedman, M. H., Hutchins, G. M., Bargeron, C. B., Deters, O. J. and Mark, F. F. (1981) Correlation between intimal thickness and fluid shear in human arteries. Atheroscler. 39, 425-436.

Kajiya, F., Tomonaga, G., Tsujioka, K., Ogasawara, Y. and Nishihara, H. (1985) Evaluation of local blood flow velocity in proximal and distal coronary arteries by laser Doppler method. J. Biomech. Engng 107, 10-15.

Ku, D. N., Giddens, D. P., Zarins, C. Z. and Glagov, S. (1985) Pulsatile flow and atherosclerosis in the human carotid bifurcation. Positive correlation between plaque location and low oscillating shear stress. Arterioscler. 5, 293-302.

Ling, S. C. and Atabek, H. B. (1972) A nonlinear analysis of pulsatile flow in arteries. J. Fluid Mech. 55, 493 $-511$.

Ling, S. C., Atabek, H. B., Fry, D. L., Patel, D. J. and Janicki, J. S. (1968) Application of heated-film velocity and shear probes to hemodynamic studies. Circ. Res. 23, 789-801.

Lou, Z. and Yang, W. J. (1991) A computer simulation of the blood flow at the aortic bifurcation. Bio-Med. Mater. Engng 1, 173-193.

Lou, Z. and Yang, W. J. (1992) Biofiuid dynamics at arterial bifurcations. Critical Rev. Biomed. Engng 19, 455-493.

Lou, Z. and Yang, W. J. (1993a) A computer simulation of the blood flow at the aortic bifurcation with flexible walls. J. Biomech. Engng (in press).
Lou, Z. and Yang, W. J. (1993b) A computer simulation of the non-Newtonian blood flow at the aortic bifurcation. $J$. Biomechanics (in press).

Lutz, R. J., Cannon, J. N., Bischoff, K. B., Dedrick, R. L., Stiles, R. K. and Fry, D. L. (1979) Shear stress patterns in a model canine artery: their relationship to atherosclerosis. in Quantitative Cardiovascular Studies: Clinical and Research Applications of Engineering Principles (Edited by Hwang, N. H. C., Gross, D. R. and Patel, D. J.) pp. 233-237. University Park Press, Baltimore.

Mark, F. F., Bargeron, C. B., Deters, O. J. and Friedman, M. H. (1989) Variations in geometry and shear rate distribution in casts of human aortic bifurcations. J. Biomechanics 22, 577-582.

McDonald, D. A. (1974) Blond Flow in Arteries. Edward Arnold, London.

Milnor, W. R. (1989) Hemodynamics. Williams \& Wilkins, Baltimore.

Pedley, T. J. (1980) The Fluid Mechanics of Large Blood Vessels. Cambridge University Press, Cambridge.

Perktold, K., Resch, M. and Florian, H. (1991) Pulsatile non-Newtonian flow characteristics in a three-dimensional human carotid bifurcation model. J. Biomech. Engng 113, 464-475.

Roache, P. J. (1972) Computational Fluid Dynamics. Hermosa Publishers. Albuquerque.

Sexl, T. (1930) Z. Phys. 61, 349-362.

Talbot, L. and Steinert, J. J. (1987) The frequency response of electrochemical wall shear probes in pulsatile flow. J. Biomech. Engng 109, 60-64.

Walburn, F. J. and Stein, P. D. (1982) The shear rate at the wall in a symmetrically branched tube simulating the aortic bifurcation. Biorheology 19, 307-316.

White, F. M. (1974) Viscous Fluid Flow. McGraw-Hill, New York.

Yamaguchi, T. and Hanai, S. (1988) To what extent does a minimal atherosclerotic plaque alter the arterial wall shear stress distribution? - a model study by an electrochemical method. Biorheology 25, 31-36. 